\title{
O Conceito de Ansiedade na Análise do Comportamento
}

\author{
The Concept of Anxiety in Behavior Analysis
}

\author{
Nilzabeth Leite Coêlho ${ }^{a}, \&$ Emmanuel Zagury Tourinho ${ }^{a}$ \\ aniversidade Federal do Pará
}

\begin{abstract}
Resumo
O conceito de ansiedade tem sido empregado na Análise do Comportamento sob controle de diferentes eventos ou relações. Neste artigo, oferecemos uma revisão dos modos como a análise do comportamento tem concebido teórica e conceitualmente o fenômeno da ansiedade e das relações que são colocadas em destaque nessas elaborações. Iniciamos com uma descrição dos usos correntes do conceito de ansiedade, assinalando que variam quanto ao papel atribuído às alterações fisiológicas, à definição das relações respondentes e operantes, verbais e não verbais, e às implicações para a terapia verbal. Discutimos, em seguida, essas variações, salientando que representam visões complementares de um fenômeno complexo, em que eventos adquirem diferentes funções a partir de processos de condicionamento direto e indireto. Finalmente, caracterizamos alguns aspectos definidores da ansiedade à luz do enfoque analítico-comportamental e argumentamos que as elaborações revisadas sugerem que a ansiedade, como problema clínico, pode guardar relação com repertórios de autocontrole.

Palavras-chave: Ansiedade; supressão condicionada; incontrolabilidade; autocontrole.
\end{abstract}

\begin{abstract}
The concept of anxiety has been used in Behavior Analysis under the control of different events or relations. In this article, we offer a theoretical and conceptual review of behavior-analytic approaches for anxiety, and of the behavioral relations that are highlighted in the literature. We start with a description of the current usages of the concept of anxiety. We point out that such usages vary from the role assigned to physiological changes, to the definition of respondent and operant relations (verbal and non-verbal) and to the implications for verbal therapy. We, then, discuss such variations as complementary explanations for a complex phenomenon, in which events acquire several functions, through processes of direct and indirect conditioning. Finally, we summarize some defining characteristics of anxiety from a behavior-analytic standpoint, and we argue that, as a clinical disorder, anxiety may be related to self-control.

Keywords: Anxiety; conditioned suppression; uncontrollability; self-control.
\end{abstract}

Conceitos científicos podem ser entendidos como respostas verbais sob controle de estímulos mais ou menos bem definidos. Entender o significado de um conceito, portanto, implica buscar as contingências das quais a resposta verbal específica é função.

O conceito psicológico de "ansiedade" tem sido apontado (e.g., Friman, Hayes \& Wilson, 1998) como impreciso, no sentido de que é empregado em sistemas explicativos diversos sob controle de eventos diferentes. Segundo Friman, Hayes et al., essa imprecisão seria favorecida pelo constante uso de metáforas, o que acaba por dificultar a construção de definições consistentes dos fenômenos para os quais os cientistas se voltam. Mesmo em um sistema explicativo avesso ao uso de metáforas, porém, o problema da imprecisão aparece. Esse é o caso da Análise do Comportamento.

Os usos do conceito de ansiedade na Análise do Comportamento têm variado em pelo menos duas direções. $\mathrm{Na}$ primeira, há uma ênfase em relações operantes não verbais

\footnotetext{
* Endereço para correspondência: Universidade Federal do Pará, Instituto de Filosofia e Ciências Humanas, Programa de Pós-Graduação em Teoria e Pesquisa de Comportamento, Rua Augusto Corrêa, 1, Guamá, Belém, PA, 66075 110. Tel.: (91) 3201 7773. E-mail: nilzabeth@gmail.com ; eztourinho@gmail.com
}

que definem o fenômeno. Na outra, a ênfase recai em relações verbais e em possíveis relações indiretas entre estímulos. Em Fester, Culbertson e Perrot Boren (1977), Lundin (1969/1977), Millenson (1967/1975) e Skinner (1953/1965, 1989), encontram-se exemplos do primeiro tipo de abordagem, enfatizando-se contingências que incluem um estímulo pré-aversivo, um estímulo aversivo (para alguns, incontrolável) e uma resposta "emocional" eliciada pelo pré-aversivo. As argumentações de Friman, Hayes et al. (1998) e Kanfer e Phillips (1974), ilustram o segundo tipo de enfoque, no qual são destacados aspectos referentes à linguagem como fonte de controle de respostas de ansiedade e relações indiretas entre estímulos (públicos e privados).

No presente trabalho, examinamos diferentes usos do conceito de ansiedade na literatura teórica, experimental e clínica da Análise do Comportamento. Buscamos, mais especificamente, oferecer uma caracterização inicial desses usos, do ponto de vista das relações comportamentais enfatizadas e da possível compatibilidade ou integração das explicações oferecidas. A análise skinneriana (cf. Estes \& Skinner, 1941/1961; Skinner, 1957, 1953/1965, 1989) é tomada como ponto de partida, visto que constitui a referência em que outros textos analítico-comportamentais se 
fundamentam. Em diferentes momentos, essa abordagem aponta que: (a) um estímulo pré-aversivo elicia respostas fisiológicas emocionais; (b) essas respostas emocionais podem elas mesmas adquirir funções aversivas; (c) um outro efeito da exposição às contingências que produzem ansiedade (estimulação aversiva com pré-sinalização) consiste da redução na taxa de resposta antes mantida por reforço positivo (a supressão condicionada); e (d) um estimulo verbal pode vir a adquirir a função eliciadora da resposta fisiológica (emocional), a partir de uma associação com o estímulo eliciador incondicionado. Nas palavras de Skinner: "há efeitos emocionais que podem ocorrer apenas quando um estímulo precede caracteristicamente um estímulo aversivo com um intervalo de tempo suficientemente grande para permitir a observação de mudanças comportamentais. A condição resultante geralmente é denominada ansiedade" (Skinner (1953/1965, p. 178).

"a condição sentida como ansiedade passa a funcionar como um segundo estímulo aversivo condicionado" (Skinner, 1989, p. 7).

"o efeito do estado emocional é uma depressão temporária da força do comportamento" (Estes \& Skinner, 1941/ 1961, p. 399).

se um estímulo verbal costuma acompanhar alguma situação, que é o estímulo não condicionado ou previamente condicionado para uma reação emocional, o estímulo verbal eventualmente evoca essa reação. Assim, se alguém tem medo de cobra e se o estímulo verbal cobra acompanhou algumas vezes cobras de verdade, o estímulo verbal, sozinho, pode evocar uma reação emocional. (Skinner, 1957, p. 154-155).

A análise que apresentamos consiste de uma revisão teórica, baseada em artigos e capítulos de livros que abordam a ansiedade sob o enfoque da Análise do Comportamento. Os textos analisados são ilustrativos de explicações analítico-comportamentais que enfatizam diferentes aspectos do fenômeno, ou diferentes relações que podem dele participar. A análise que oferecemos inicia com uma descrição dos usos do conceito de ansiedade naquela literatura, com destaque para as referências a: mudanças fisiológicas, relações comportamentais (respondentes, operantes, verbais, não verbais), operações estabelecedoras e implicações para a terapia verbal. Na segunda parte, discutimos aqueles usos do conceito de ansiedade destacando sua compatibilidade e sua possível integração em uma explicação analíticocomportamental para o fenômeno.

\section{Usos do Conceito de Ansiedade na Análise do Comportamento}

Os diferentes usos do conceito de ansiedade ilustrados nos trabalhos examinados envolvem a referência a componentes fisiológicos, condicionamento operante e respondente, direto e indireto, não verbal e verbal, além de relações com operações estabelecedoras e a terapia verbal.

\section{Ansiedade e Mudanças Fisiológicas}

A imprecisão com que a Análise do Comportamento tem definido as fronteiras entre seu objeto de estudo e a fisiolo- gia dos organismos (cf. Reese, 1996a, 1996b) encontra-se exemplificada de modo singular na literatura que, sob um enfoque analítico-comportamental, discute a ansiedade. Invariavelmente, definições dos fenômenos tidos como instâncias de ansiedade fazem referência ao que se passa com a fisiologia do organismo. O lugar das mudanças fisiológicas em uma concepção analítico-comportamental da ansiedade, todavia, ora passa sem uma discussão, ora conduz a proposições não coincidentes.

A exposição do organismo a estímulos aversivos e préaversivos, controláveis ou incontroláveis, produz uma condição fisiológica particular, concomitante a uma mudança no responder geral do organismo. Alguns textos analisados (e.g., Forsyth, 2000; Forsyth \& Eifert, 1996a, 1996b; Oliveira \& Duarte, 2004; Queiroz \& Guilhardi, 2001; Reiss, Peterson, Gursky \& McNally, 1986; Wolpe, 1981) focalizam especificamente as contingências sob as quais essa condição fisiológica é produzida. Queiroz e Guilhardi, por exemplo, assinalam que "a ansiedade é um estado corporal produzido por contingências de reforçamento específicas: um estímulo sinaliza a apresentação de um estímulo aversivo e não há comportamento de fuga-esquiva possível" (p. 257).

Algumas vezes, a ênfase na condição fisiológica é tal, que as contingências que a produzem ficam em um segundo plano nas interpretações oferecidas (cf. Barlow, Rapee \& Brown, 1992; Zettle, 2003). Em outros casos (e.g., Bornstein, 1975; Santos, 2000; Wlazlo, Hartwing, Hand, Kaiser \& Münchau, 1990; Wolpe, 1981), a condição fisiológica é um mero "sintoma" de um fenômeno que, nesse caso, é necessariamente algo diferente, embora nem sempre esclarecido.

Em alguns trabalhos, a alteração fisiológica produzida pelas contingências que explicam a ansiedade é abordada salientando-se suas funções em uma relação comportamental. Nesses casos, a condição fisiológica pode ser vista como: (a) um estímulo eliciador de respostas verbais ou não verbais; (b) um estímulo discriminativo para respostas não verbais mantidas por reforço negativo; e (c) um estímulo discriminativo para respostas não verbais mantidas por reforço positivo. Portanto, nos casos em que a condição fisiológica adquire uma função, temos uma inter-relação entre as contingências que produziram a condição fisiológica e essas novas relações das quais a condição fisiológica participa. Forsyth, Eifert e Thompson (1996), por exemplo, assinalam que "como resultado de uma associação entre dicas internas ... e falsos alarmes, essas dicas internas podem em seguida evocar respostas de alarme aprendidas" (p. 392-393).

Ansiedade como Relações Respondentes e Operantes Não Verbais Versus Relações Respondentes e Operantes Verbais, Versus Relações Respondentes e Operantes Verbais e Não Verbais

Quando a ansiedade é interpretada sob a ótica de relações de contingência, três enfoques prevalecem: relações respondentes e operantes não verbais, relações respondentes e operantes verbais e relações respondentes e operantes verbais e não verbais. Em todos os casos, componentes 
operantes constituem o foco principal e assumem formas mais variadas. Componentes respondentes limitam-se à eliciação da resposta fisiológica pelo estímulo pré-aversivo ou por estímulos verbais a ele associados.

Relações Respondentes e Operantes Não Verbais. Efeitos diversos da exposição a um estímulo pré-aversivo sobre o responder não verbal são discutidos na literatura examinada. Um primeiro efeito é a redução do responder mantido por reforço positivo, efeito designado de supressão condicionada (cf. Forsyth, 2000; Oliveira \& Duarte, 2004; Queiroz \& Guilhardi, 2001; Sanger \& Blackman, 1976; Silva, 1997; Viliers \& Millenson, 1972; Zamignani \& Banaco, 2005).

Se houver a possibilidade de emissão das respostas de fuga do estímulo condicionado e/ou fuga do incondicionado, essas respostas tornam-se mais prováveis de serem emitidas do que as que levariam à produção de estímulos reforçadores positivos. Caso não haja a possibilidade de respostas de fuga e esquiva, o efeito reflexo da estimulação condicional paralisa a emissão de respostas operantes que produzem o estímulo reforçador positivo. A esta descrição da ansiedade deuse o nome de 'supressão condicionada.' (Zamignani \& Banaco, 2005, p. 83).

Um segundo efeito é a aquisição de respostas de fuga e/ ou esquiva da estimulação aversiva (cf. Barbosa, 2004; Bouton, Mineka \& Barlow, 2001; Cone, 1998; Eifert, 1984; Eifert \& Wilson, 1991; Forsyth, 2000; Friman, Hayes et al., 1998; Hopko, McNaiel, Zvolensky \& Eifert, 2001; Jones \& Friman, 1999; Lejuez, O’Donnell, Wirth, Zvolensky, \& Eifert, 1998; Oliveira \& Duarte, 2004; Öst, 1987; Reiss et al., 1986; Santos, 2000; Silva, 1997; Zamignani \& Banaco, 2005; Zamignani \& Vermes, 2003; Wlazlo et al., 1990; Wolpe, 1981). Quando a aquisição de respostas de fuga/ esquiva é referida, isso significa uma interpretação da ansiedade de acordo com a qual a estimulação aversiva pré-sinalizada é evitável, isto é, controlável. Nesse caso, há uma resposta capaz de evitá-la ou suprimi-la. Segundo Barbosa, "as pessoas ansiosas tendem a esquivar-se de situações que evocam a ansiedade. A esquiva é um comportamento natural dos organismos resultante dos reforçadores que são amplamente liberados pelo contexto sócioverbal" (p. 164).

Para alguns autores (Forsyth \& Eifert, 1996a; Zvolensky, Lejuez \& Eifert, 1998), porém, a estimulação aversiva na ansiedade nem sempre é evitável, ou controlável. A uma variação em sua controlabilidade, por outro lado, pode corresponder uma variação (aumento ou redução) da ansiedade. Segundo Zvolensky et al., "tem sido postulado que uma falta de controle sobre eventos aversivos internos (fisiológicos) e externos (ambientais) desempenha um papel central no desenvolvimento e manutenção de muitos transtornos de ansiedade" (p. 193-194).

Mudanças no responder operante não verbal podem ainda estar relacionadas a um reforçamento positivo ou negativo do responder ansioso (cf. Zamignani \& Banaco, 2005; Woods \& Miltenberger, 1996). Zamignani e Banaco, por exemplo, assinalam que a ansiedade pode ser mantida pela suspensão ou adiamento de tarefas indesejáveis, ou pela obtenção de atenção social (cf. Zamignani \& Banac, 2005; Zamignani \& Vermes, 2003). Zamignani e Vermes apontam que "a função de esquiva não é a única possível na manutenção de um transtorno de ansiedade e as conseqüências que mantém o problema podem ser mais diversas, envolvendo diferentes configurações de contingências" (p. 118).

Relações Operantes Verbais. Naqueles trabalhos que se voltam para relações operantes verbais na análise da ansiedade, a discussão focaliza o condicionamento semântico (cf. Bouton et al., 2001; Eifert, 1984; Forsyth \& Eifert, 1996b; Forsyth, Eifert \& Thompson, 1996; Tyndall, Roche \& James, 2004; Weiss \& Evans, 1978), ou o processo de formação de classes de estímulos equivalentes (cf. Forsyth \& Eifert, 1996a; Friman, Hayes et al., 1998; Friman, Wilson \& Hayes, 1998; Tyndall et al., 2004; Zamignani \& Banaco, 2005), por meio dos quais palavras adquirem funções aversivas condicionadas, integrando o fenômeno emocional para um indivíduo. Na perspectiva do condicionamento semântico, Eifert assinala que "se estímulos verbais incondicionados ... são freqüentemente pareados com a visão de uma cobra ou com a palavra 'cobra', então a palavra imagem ou visão de uma cobra levarão a respostas emocionais (fisiológicas) negativas" (p. 14). Friman, Hayes et al. discutem a formação de classes de estímulos equivalentes, incluindo estímulos verbais, no controle de respostas ansiosas:

Para pessoas ansiosas com habilidades verbais, os relatos de ansiedade ... podem também ser reativos e, assim, gerar efeitos indesejados. Isto é, eles não apenas descrevem o comportamento da pessoa e suas circunstâncias, mas podem também alterar a função do comportamento e das circunstâncias descritas. (p. 144-145).

Em uma outra direção, estímulos verbais podem evitar respostas ansiosas. Isto é "autoverbalizações", respostas verbais emitidas por um indivíduo, que entram no controle de seu comportamento subseqüente, podem funcionar para controlar respostas de ansiedade (cf. Cone, 1998; Eifert \& Wilson, 1991; Hayes, Hussian, Turner, Anderson \& Grubb, 1983; Lejuez et al., 1998; Zamignani \& Banaco, 2005). Segundo Cone, "ao ensinar o cliente a falar de modo diferente ... o terapeuta o ajuda a reassumir a responsabilidade por suas circunstâncias" (p. 330).

Relações Operantes Verbais e Não Verbais. Em alguns contextos, relações respondentes e operantes, verbais e não verbais são enfocadas na análise da ansiedade (cf. Bouton et al., 2001; Cone, 1998; Eifert, 1984; Eifert \& Wilson, 1991; Forsyth, 2000; Forsyth \& Eifert, 1996a, Friman, Hayes et al., 1998; Friman, Wilson et al., 1998; Hopko et al., 2001; Tyndall et al., 2004; Zamignani \& Banaco, 2005). As abordagens oferecidas parecem, nesses casos, apreender dimensões mais amplas do fenômeno, em particular no grau de complexidade que ele alcança para humanos e que se estende para além das contingências investigadas origi- 
nalmente por Estes e Skinner (1941/1961), incluindo processos verbais diversos. Contrapondo sua análise àquela de Skinner, Friman, Hayes et al. afirmam que

as respostas de pessoas com habilidades verbais em circunstâncias similares são diferentes daquelas de organismos mais simples, como ratos. Para o humano, o choque [estímulo aversivo empregado nos experimentos de ansiedade com ratos] e o relato tendem a relacionar-se bidirecionalmente e, assim, a compartilhar funções. (p. 144).

Em que pese a maior abrangência dos trabalhos que abordam os tipos diversos de relações que podem vir a compor uma instância de ansiedade, trata-se de esforços que não oferecem uma integração das diversas propostas que veiculam. Como resultado, permanecemos sem uma sistematização ampla dos fenômenos em graus variáveis de complexidade sob controle dos quais analistas do comportamento falam em ansiedade.

\section{Relações Operantes Verbais Versus Operações Estabelecedoras}

Componentes verbais admitidos como constitutivos de fenômenos de ansiedade em algumas circunstâncias são interpretados não como estímulos eliciadores da resposta fisiológica, nem como estímulos discriminativos para respostas de fuga/esquiva, mas como eventos que alteram a responsividade do organismo a certas contingências, ou operações estabelecedoras (cf. Reiss et al., 1986; Torres, 2000; Wlazlo et al., 1990; Wolpe, 1981). Reiss et al. apontam que a

sensibilidade à ansiedade [a crença de que a experiência de ansiedade/medo causa doença] aumenta a atenção a estímulos que sinalizam a possibilidade de ficar ansioso, aumenta a preocupação com a possibilidade de ficar ansioso e aumenta a motivação para evitar estímulos provocadores de ansiedade. (p. 2).

Por outro lado, Zamignani e Banaco (2005) fazem referência a operações estabelecedoras constitutivas da ansiedade não necessariamente verbais. Por exemplo, mencionam contingências aversivas que levam o indivíduo a uma condição crônica de interações, tornando mais provável a ansiedade. Também o estado de privação é visto como uma operação estabelecedora que participa da ansiedade. Se há poucos reforçadores positivos (especialmente sociais) disponíveis no ambiente, a responsividade a contingências que levam a sua produção será maior, ainda que isso implique relações típicas da ansiedade.

\section{Implicações para a Terapia Verbal}

Os tipos de relações consideradas constitutivas do fenômeno comportamental da ansiedade em cada um dos artigos analisados repercutem diretamente nos tipos de intervenção propostos. Novamente, como os trabalhos tendem a focalizar aspectos diversos, o que aparece como recomendação de intervenção não constitui um corpo de técnicas e procedimentos consensuais.

Dada a centralidade do componente fisiológico, conforme citado anteriormente, em diversos textos (cf. Barlow et al., 1992; Bornstein, 1975; Bouton et al., 2001; Forsyth, 2000; Forsyth \& Eifert, 1996a; Öst, 1987, 1988; Peterson, 1995; Zettle, 2003) encontra-se a recomendação de uma intervenção que vise principalmente a modificação das alterações fisiológicas. Segundo Öst (1987), "uma boa maneira de interromper esse desenvolvimento é focalizar as reações fisiológicas e aprender a não responder tão fortemente" (p. 398).

Há, por outro lado, propostas de intervenção orientadas pela compreensão dos diversos componentes operantes que podem definir a ansiedade para um indivíduo. Nesses casos, são discutidas as diversas funções que respostas de ansiedade podem ter na vida do indivíduo (e.g., Barbosa, 2004; Santos, 2000; Zamignani \& Banaco, 2005). Zamignani e Banaco, 2005; Zamignani e Vermes, 2003 e Wlazlo et al., 1990), ressaltam a importância de um levantamento e aquisição de novas habilidades relacionadas a déficits comportamentais. Queiroz e Guilhardi (2001) ressaltam a necessidade da modelagem de uma resposta de fuga/esquiva possível para o tratamento e diminuição da ansiedade. Em uma outra direção, há trabalhos que sugerem a importância de mudar as contingências (aversivas) ambientais envolvidas na instalação e manutenção da ansiedade (cf. Cone, 1998; Queiroz \& Guilhardi, 2001; Santos, 2000; Zamignani \& Vermes, 2003). Torres (2000), por outro lado, sugere abordar os componentes verbais envolvidos na ansiedade promovendo um enfraquecimento do contexto de literalidade (contingências sócio-verbais que colocam o responder verbal - e.g., autodescritivo - sob controle de eventos específicos). Mais recentemente, a Terapia de Aceitação e Compromisso (ACT) (cf. Barbosa; Cone; Forsyth, 2000; Torres; Zettle, 2003) e a Terapia Analítica Funcional (FAP) (cf. Barbosa; Zamignani \& Vermes) têm sido apontadas como terapias eficazes no tratamento de ansiedade.

Barbosa (2004) sugere ainda a possibilidade de um acompanhamento multidisciplinar para o tratamento de ansiedade. Forsyth (2000), Zinbarg (1993) e Wolpe (1977, 1981), enfatizam técnicas utilizadas pela Terapia Cognitiva.

Em Barlow et al. (1992), Bouton et al. (2001), Friman, Hayes et al. (1998) e Wolpe (1977) encontra-se a recomendação de que o tratamento de ansiedade, para ser mais eficaz, deve ser realizado pela combinação de pelo menos dois tipos de intervenção (que focalizem contingências verbais e não verbais). Friman, Hayes et al., por exemplo, falam da combinação de técnicas de exposição ao estímulo aversivo com uma abordagem de componentes verbais da ansiedade:

Para ser completamente efetiva, a exposição freqüentemente pode ter que incluir todos, ou pelo menos um número maior dos eventos que funcionalmente ocasionam a esquiva mal adaptativa. A maioria das pessoas ansiosas procura tratamento para lidar com a presença do objeto ou evento temido, enquanto não pensam ou sentem medo. Isso significa que o aspecto verbal do medo é parte do evento temido. (p. 150). 


\section{Discutindo os Usos do Conceito de Ansiedade na Análise do Comportamento: (In)Compatibilidade e Integração}

Como assinalado na introdução deste artigo, nosso objetivo é analisar os diferentes usos do conceito de ansiedade identificando os tipos de relações sugeridas e a possível integração entre as explicações oferecidas. Nessa direção, buscamos sistematizar, nos parágrafos seguintes, os aspectos que controlam as descrições dos autores, ou seja, os eventos ou relações de contingências sob controle dos quais são oferecidas suas interpretações.

Uma primeira observação é a de que os textos examinados referem-se à ansiedade sob controle de aspectos como: (a) as relações comportamentais envolvidas; (b) as contingências que produzem aquelas relações; (c) as condições corporais produzidas concomitantemente pelas mesmas contingências; (d) as funções dessas condições corporais nas relações comportamentais; (e) os processos por meio dos quais estímulos verbais participam dessas relações; (f) os ambientes sociais que favorecem a instalação e manutenção de ansiedade etc.

$\mathrm{O}$ aspecto principal abordado diz respeito à presença de contingências aversivas, tanto na instalação quanto na manutenção da ansiedade. Desde os modelos experimentais, passando pela definição de supressão condicionada, ou pela importância das respostas de fuga e esquiva da estimulação aversiva, a sinalização do estímulo aversivo pelo estímulo pré-aversivo é tida como uma contingência importante na conceituação da ansiedade.

Embora a literatura enfatize contingências em que um evento sinaliza previamente um estímulo aversivo, há circunstâncias na vida cotidiana em que um evento sinaliza previamente um estímulo reforçador positivo e um tipo de ansiedade parece emergir. A sinalização de uma viagem muita esperada, ou de um presente desejado constituem exemplos dessas circunstâncias, quando tal sinalização acaba por adquirir funções aversivas, relacionadas à espera. Segundo Zamignani e Banaco (2005), esse tipo de contingência não é o que dá origem à ansiedade como problema clínico. Mas, nesse caso, estamos diante de uma situação em que a literatura (teórica, experimental e clínica) tende a enfatizar um aspecto (a função aversiva do evento sinalizado) por sua relevância clínica, ignorando uma variação relevante no fenômeno simplesmente porque ela não dá (pelo menos com a mesma freqüência) origem a demandas terapêuticas. Por outro lado, ainda é necessário explicar por quê a sinalização de um reforçador positivo, mesmo assumindo uma função aversiva (a sinalização), não dá origem aos mesmos problemas.

Se um evento sinaliza um estímulo atrasado, mas não elicia a chamada resposta fisiológica de ansiedade, nem o indivíduo pára de responder sob controle de outras contingências, isso provavelmente significa que "aprendeu a esperar". Na nossa cultura, essa aprendizagem se realiza freqüentemente sob a forma de uma espera por reforços positivos, e no contexto de contingências de autocontrole. Nesse caso, possivelmente a ansiedade guarda relação com repertórios de autocontrole, em particular, a "ansiedade clínica" (que envolve a espera por estímulos aversivos). Se o indivíduo, em sua história de vida, já aprendeu a responder sob controle de reforços atrasados de maior magnitude (autocontrole), em contextos de esquemas concorrentes, pode ser menos provável que a sinalização do estímulo atrasado assuma funções aversivas. Entretanto, para um indivíduo que responde predominantemente sob controle de contingências imediatas (impulsividade) será mais provável que sinalização do reforço atrasado se torne um evento com funções aversivas, capaz de gerar ansiedade. Se entendermos que problemas com o autocontrole podem estar relacionados com a ansiedade como queixa clinica, mesmo que em menor medida, a análise dessas variáveis se torna ainda mais importante.

Um outro aspecto a ser considerado diz respeito à incontrolabilidade sobre a apresentação do estímulo aversivo sinalizado. Tem sido apontado que uma história de incontrolabilidade dá origem ao desamparo aprendido, a "dificuldade de aprendizagem apresentada por indivíduos que tiveram experiência prévia com estímulos incontroláveis" (Hunziker, 2005, p. 131). O desamparo aprendido, por seu turno, tem sido considerado um modelo animal da depressão (cf. Hunziker). Nesse caso, o que torna ansiedade e depressão conceitual e praticamente diferentes?

$\mathrm{Na}$ ansiedade, diferente da depressão, há uma sinalização dos estímulos incontroláveis, em geral estímulos aversivos, daí falar-se da sinalização como estímulo pré-aversivo. A estimulação pré-aversiva também adquire funções aversivas. Assim, quando há incontrolabilidade na ansiedade, esse componente está associado à pré-sinalização, o que não constitui uma condição necessária para a produção do desamparo aprendido (e, assim, da depressão).

Tanto quanto a sinalização, as respostas fisiológicas eliciadas pelo estímulo sinalizador constituem o núcleo das abordagens analisadas (exceto para Barlow et al., 1992, e para Zettle, 2003). Ambos, estímulo sinalizador e resposta fisiológica, adquirem funções aversivas para os organismos. A função da resposta fisiológica, porém, é variável, dependendo do enfoque oferecido. Ela pode ser entendida como nada mais do que subproduto das contingências, como uma resposta do organismo (eliciada pelo pré-aversivo e, posteriormente, por estímulos associados/equivalentes), como um estímulo discriminativo para autodescrições de ansiedade, como estímulo discriminativo para respostas não verbais de fuga/esquiva mantidas por reforçamento negativo, como estímulo discriminativo para respostas não verbais mantidas por reforçamento positivo e mesmo como uma condição estabelecedora, afetando a responsividade do organismo a outras contingências, inclusive de reforçamento positivo. Em qualquer circunstância, importa ressaltar que a resposta fisiológica é sempre parte do fenômeno da ansiedade, ou seja, a ansiedade não estaria circunscrita a essa ocorrência, sendo, portanto algo que vai além disso. Nesse caso, seria possível uma apreciação da ansiedade que integre componentes respondentes e operantes dos fenômenos emocionais, como ilustrado em Darwich e Tourinho (2005). 
Considerando, novamente, as possíveis relações entre a resposta fisiológica de ansiedade e componentes operantes verbais, tem-se que as primeiras podem vir a ser eliciadas por estímulos verbais a partir de processos diversos, em particular, do condicionamento semântico e da formação de classes de estímulos equivalentes. Uma maior atenção a esses processos verbais tem tornado evidente a necessidade de um modelo de interpretação dos fenômenos emocionais em geral, e da ansiedade em particular, que considere as diversas relações interconectadas que os constituem.

Tourinho (2006) propõe um tipo de análise para os fenômenos emocionais que tenta dar conta de sua complexidade variável ao longo de um continuum, considerando relações produzidas no nível filogenético, ontogenético e cultural. A noção de um continuum de complexidade afirma que os fenômenos comportamentais podem ser interpretados de acordo com o grau de complexidade em que se apresentam as diversificadas relações envolvidas. Deste ponto de vista, fenômenos dos quais participam apenas relações de origem filogenética apresentar-se-iam como menos complexos do que aqueles dos quais participam também relações produzidas pelo condicionamento respondente e operante; estes, por seu turno, seriam menos complexos ainda do que aquelas dos quais participam adicionalmente relações que têm origem em um nível cultural. Entendendo sob este enfoque, a ansiedade poderia apresentar-se sob a forma de um evento de maior ou menor complexidade dependendo dos tipos de relações envolvidas.

Quando se trata dos tipos de intervenção pode-se perceber que elas enfatizam aspectos diferentes dessas relações. Algumas intervenções enfatizam aspectos relacionados à condição corporal, visando principalmente uma redução nos sintomas fisiológicos, a fim de garantir uma diminuição da ansiedade. Outras propostas de intervenção são realizadas enfocando as relações operantes não verbais envolvidas na definição de ansiedade. São intervenções realizadas objetivando, principalmente, modificações ambientais e/ou comportamentais relacionadas com a contingência aversiva. Por último, há as intervenções que visam também uma modificação nas relações operantes verbais envolvidas na ansiedade. Neste caso, as intervenções têm como objetivo principal alterar a função dos eventos verbais como eliciadores da condição corporal característica de ansiedade.

À luz do modelo proposto por Tourinho (2006), pode-se interpretar esses diferentes tipos de intervenção como possibilidades de alcance da terapia analítico-comportamental. Dessa maneira, se entendemos a ansiedade como um fenômeno comportamental com diferentes níveis de complexidade, as intervenções propostas deveriam visar as diferentes relações que se apresentam na definição do fenômeno. Nesse sentido, não se trata de afirmar que uma intervenção é mais eficaz do que outra, mas sim de elucidar que se deve intervir sobre as relações que estão envolvidas, sendo elas dos níveis filogenético, ontogenético ou cultural.

\section{Síntese e Conclusão}

As variações de definição encontradas para o conceito de ansiedade na literatura analítico-comportamental não são necessariamente incompatíveis, na medida em que abordam diferentes relações de um fenômeno complexo. O que há de comum nas várias definiçõos examinadas é a importância atribuída a uma sinalização do estímulo aversivo pelo estímulo pré-aversivo e sua função na eliciação de respostas fisiológicas. A partir disso, redes de relações mais ou menos complexas são construídas e vêm a definir a ansiedade de um indivíduo. Assim, autores que referem apenas componentes respondentes o fazem sob controle de um aspecto da ansiedade; o mesmo pode ser dito dos que se limitam aos componentes operantes não verbais ou verbais.

Os diferentes usos do conceito de ansiedade discutidos neste trabalho correspondem, portanto, a recortes diferenciados de um mesmo fenômeno. A idéia de que esse fenômeno varia ao longo de um continuum de complexidade, e de que essa complexidade variável se define pela participação de relações interconectadas, produzidas por diferentes tipos de variáveis, pode ser útil para o planejamento da intervenção.

A presente análise acrescenta, ainda, que um componente das relações que definem a ansiedade em seus diferentes graus de complexidade é a sinalização de um evento com função reforçadora negativa ou positiva, que pode adquirir uma função aversiva. O evento sinalizador é, por isso, chamado de estímulo pré-aversivo (embora possa ser pré-reforço positivo). Além disso, o estímulo pré-aversivo elicia condições fisiológicas específicas e reduz a taxa de respostas mantida por reforço positivo. Essa condição fisiológica pode ter uma função estabelecedora, "imobilizando" o indivíduo e afetando assim sua responsividade a contingências ambientais. Entretanto, o efeito paralisante da sinalização de um evento aversivo (redução da taxa de resposta) pode ser maior do que o de um evento reforçador positivo, daí apenas o primeiro dar origem à queixa clínica.

Deve-se considerar também que o efeito aversivo condicionado do evento sinalizador pode depender de o indivíduo já ter adquirido repertórios de autocontrole, caracterizados por um responder sob controle de reforços atrasados, de maior magnitude. O autocontrole talvez explique porque é mais provável que o sinalizador de evento reforçador negativo assuma funções aversivas condicionadas. No autocontrole, aprendemos a responder sob controle de eventos positivos atrasados mesmo que existam conseqüências negativas imediatas, mas não sob controle de eventos aversivos atrasados.

Outro ponto examinado diz respeito à incontrolabilidade de um evento aversivo poder acontecer com a sinalização pré-aversiva, como no caso da ansiedade, ou sem a sinalização pré-aversiva, como é o caso da depressão (cf. Cavalcante, 1997), sendo que quando há o pré-aversivo, ele próprio adquire funções aversivas. Desta forma, a ocorrência da sinalização do estímulo pré-aversivo, na ansiedade, confere ao fenômeno uma dimensão de previsibilidade. A 
inexistência de tal sinalização nas relações que definem a depressão confere a esta um componente de imprevisibilidade. As implicações para a intervenção em cada caso merecem ainda ser examinadas.

A supressão condicionada aparece sob a forma de não aprender a responder sob contingências em que a resposta poderia produzir reforçamento negativo (depressão) e de não emitir uma resposta anteriormente aprendida, que pode produzir reforços positivos, quando exposto a um estímulo pré-aversivo (ansiedade). Em ambos os casos a condição fisiológica pode ter uma função estabelecedora (supressora) condicionada. Essa mesma função estabelecedora (supressora) pode vir a ser adquirida por estímulos associados à condição fisiológica, como, por exemplo, as autodescrições.

Provavelmente, a escassez de trabalhos conceituais e empíricos focalizando a ansiedade sob a ótica da Análise do Comportamento decorre da diversidade dos arranjos de relações que podem definir o fenômeno, da possibilidade de abordá-lo a partir de cada um desses arranjos e da correspondente variedade de intervenções.

À luz dessa sistematização, uma agenda de pesquisas sobre a ansiedade na Análise do Comportamento poderia focalizar questões como: (a) o papel da sinalização de reforçamento positivo e (b) a relação entre repertórios de autocontrole e ansiedade.

\section{Referências}

Barbosa, C. (2004). Ansiedade: Possíveis intervenções na Análise do Comportamento. In M. Z. S. Brandão, F. C. S Conte, F. C. Brandão, Y. K. Ingberman, V. M. Silva \& S. O. Oliane (Eds.), Sobre comportamento e cognição: Vol. 13 (pp. 163-167). Santo André, SP: ESEtec.

Barlow, D. H., Rapee, R. M., \& Brown, T. A. (1992). Behavioral treatment of generalized anxiety disorder. Behavior Therapy, 23, 551-570.

Bornstein, P. H. (1975). Innovation in technique: A group-based induced anxiety. Revista Mexicana de Analisis de la Conducta, 1, 299-301.

Bouton, M. E., Mineka, S., \& Barlow, D. H. (2001). A modern learning theory perspective on the etiology of panic disorder. Psychological Review, 108, 4-32.

Cavalcante, S. N. (1997). Notas sobre o fenômeno depressão a partir de uma perspectiva analítico-comportamental. Psicologia: Ciência e Profissão, 17, 2-12.

Cone, J. D. (1998). Hierarchical views of anxiety: What do they profit us? Behavior Therapy, 29, 325-332.

Darwich, R. A., \& Tourinho, E. Z. (2005). Respostas emocionais à luz do modo causal de seleção por conseqüências. Revista Brasileira de Terapia Comportamental e Cognitiva, 7, 107118.

Eifert, G. H. (1984). The effects of language conditioning on various aspects of anxiety. Behavior Research and Therapy, $22,13-21$.

Eifert, G. H., \& Wilson, P. H. (1991). The triple response approach to assessment: A conceptual and methodological reappraisal. Behavior Research and Therapy, 29, 283-292.

Estes, W. K., \& Skinner, B. F. (1961). Some quantitatives properties of anxiety. In B. F. Skinner (Ed.), Cumulative record: Enlarged Edition (2. ed., pp. 393-404). New York: AppletonCentury-Crofts. (Original publicado em 1941)
Fester, C. B., Culbertson, S., \& Perrot Boren, M. C. (1977). Princípios do comportamento. São Paulo, SP. Hucitec.

Forsyth, J. P. (2000). A process-oriented behavioral approach to the etiology, maintenance, and treatment of anxiety - Related disorders. In M. J. Dougher (Ed.), Clinical behavior analysis (pp. 153-180). Reno, NV: Context Press.

Forsyth, J. P., \& Eifert, G. H. (1996a). Systematic alarms in fear conditioning I: A reappraisal of what is being conditioned. Behavior Therapy, 27, 441-462.

Forsyth, J. P., \& Eifert, G. H. (1996b). The language of feeling and the feeling of anxiety: Contributions of the behaviorisms toward understanding the function-altering effects of language. The Psychological Record, 46, 607-649.

Forsyth, J. P., Eifert, G. H., \& Thompson, R. N. (1996). Systematic alarms in fear conditioning II: An experimental methodology using $20 \%$ carbon dioxide inhalation as an unconditioning stimulus. Behavior Therapy, 27, 391-415.

Friman, P. C., Hayes, S. C., \& Wilson, K. G. (1998). Why behavior analysts should study emotion: The example of anxiety. Journal of Applied Behavior Analysis, 31, 137-156.

Friman, P. C., Wilson, K. G., \& Hayes, S. C. (1998). Behavior analysis of private events is possible, progressive, and nondualistic: A response to Lamal. Journal of Applied Behavior Analysis, 31, 707-708.

Hayes, S. C., Hussian, R. A., Turner, A. E., Anderson, N. B., \& Grubb, T. D. (1983). The effects of coping statements on progress through a desensitization hierarchy. Journal of Behavior Therapy and Experimental Psychiatry. 14, 117-129.

Hopko, D. R., McNaiel, D. W., Zvolensky, M. J., \& Eifert, G. H. (2001). The relation between anxiety and skill in performancebased anxiety disorders: A behavioural formulation of social phobia. Behavior Therapy, 32, 185-207.

Hunziker, M. H. L. (2005). O desamparo aprendido revisitado: Estudos com animais. Psicologia: Teoria e Pesquisa, 21, 131139.

Jones, K. M., \& Friman, P. C. (1999). A case study of behavioral assessment and treatment of insect phobia. Journal of Applied Behavior Analysis, 32, 95-98.

Kanfer, F. H., \& Phillips, J. S. (1974). Os princípios da aprendizagem na terapia comportamental. São Paulo, SP: EPU.

Lejuez, C. W., O’Donnell, J., Wirth, O., Zvolensky, M. J., \& Eifert, G. H. (1998). Avoidance of $20 \%$ carbon dioxide-enriched air with humans. Journal of the Experimental Analysis of behavior, 70, 79-86

Lundin, R. W. (1977). Personalidade: Uma análise do comportamento (R. R. Kerbauy, Trad.). São Paulo, SP: EPU. (Original publicado em 1969)

Millenson, J. R. (1975). Princípios de análise do comportamento. Brasília, DF: Coordenada. (Original publicado em 1967)

Oliveira, M. A., \& Duarte, A. M. M. (2004). Controle de respostas de ansiedade em universitários em situações de exposições orais. Revista Brasileira de Terapia Comportamental e Cognitiva, 6, 183-199.

Öst, L. G. (1987). Applied relaxation: Description of a coping technique and review of controlled studies. Behavior Research and Therapy, 25, 397-409.

Öst, L. G. (1988). Applied relaxation vs progressive relaxation in the treatment of panic disorder. Behavior Research and Therapy, 26, 13-22.

Peterson, L. (1995). Special series: Mechanisms, populations, and treatments innovations in anxiety disorders. Behavior Therapy, 26, 451-455. 
Queiroz, P. P., \& Guilhardi, H. J. (2001). Identificação e análise de contingências geradoras de ansiedade: Caso clínico. In H. J. Guilhardi, M. B. B. P. Madi, P. P. Queiroz \& M. C. Scoz (Eds.), Sobre comportamento e cognição: Vol. 7 (pp. 257-268). Santo André, SP: ESETec.

Reese, H. W. (1996a). How is physiology relevant to behavior analysis? The Behavior Analyst, 19, 61-70.

Reese, H. W. (1996b). Response to commentaries. The Behavior Analyst, 19, 85-88.

Reiss, S., Peterson, R. A., Gursky, D. M., \& McNally, R. J. (1986). Anxiety sensitivity, anxiety frequency and the prediction of fearfulness. Behavior Research and Therapy, 24, 1-8.

Sanger, D. J., \& Blackman, D. E. (1976). The effects of dAmphetamine on the temporal control of operant responding in rats during a preshock stimulus. Journal of the Experimental Analysis of Behavior, 26, 369-378.

Santos, A. M. (2000). Modelo comportamental da ansiedade. In R. R. Kerbauy (Ed.), Sobre comportamento e cognição: Vol. 5 (pp. 189- 191). Santo André, SP: SET.

Silva, M. T. A. (1997). Modelos animais de ansiedade. In D. R. Zamignani (Ed.), Sobre comportamento e cognição: Vol. 3 (pp. 91-96). Santo André, SP: ARBytes.

Skinner, B. F. (1957). Verbal behavior. New York: AppletonCentury.

Skinner, B. F. (1965). Science and human behavior. New York: Free Press. (Original publicado em 1953)

Skinner, B. F. (1989). Recent issues in the analysis of behavior. Columbus, $\mathrm{OH}$ : Merrill.

Torres, N. (2000). Ansiedade: O enfoque do behaviorismo radical respaldando procedimentos clínicos. In R. C. Wielenska (Ed.), Sobre comportamento e cognição: Vol. 6 (pp. 228-238). Santo André, SP: ARBytes.

Tourinho, E. Z. (2006). Private stimuli, covert responses and private events: Conceptual remarks. The Behavior Analyst, 29, 13-31.

Tyndall, I. T., Roche, B., \& James, J. E. (2004). The relation between stimulus function and equivalence class formation. Journal of the Experimental Analysis of Behavior, 81, 257266.

Viliers, P. A., \& Millenson, J. R. (1972). Concurrent performances: A baseline for the study of conditioned anxiety. Journal of the Experimental Analysis of Behavior, 18, 287-294.

Zamignani, D. R., \& Banaco, R. A. (2005). Um panorama analítico-comportamental sobre os transtornos de ansiedade. Revista Brasileira de Terapia Comportamental e Cognitiva, 7, 77-92.

Zamignani, D. R., \& Vermes, J. S. (2003). Propostas analíticocomportamentais para o manejo de transtornos de ansiedade: Análise de casos clínicos. In H. M. Sadi \& N. M. S. Castro. (Eds.), Ciência do comportamento. Conhecer e avançar: Vol. 3 (pp. 117-135). Santo André, SP: ESETec.

Zettle, R. D. (2003). Acceptance and commitment therapy (ACT) vs systematic desensitization in treatment of mathematics anxiety. The Psychological Record, 53, 197-215.

Zinbarg, R. E. (1993). Information processing and classical conditioning: Implications for exposure therapy and the integra-tion of cognitive therapy and behavior therapy. Journal of Behavior Therapy and Experimental Psychiatry, 24, 129-139.

Zvolensky, M. J., Lejuez, C. W., \& Eifert, G. H. (1998). The role of offset control in anxious responding: An experimental test using repeated administrations of $20 \%$ carbon dioxide-enriched air. Behavior Therapy, 29, 193-209.
Weiss, A. R., \& Evans, I. M. (1978). Process studies in language conditioning - I: Counterconditioning of anxiety by "calm" words. Journal of Behavior Therapy and Experimental Psychiatry, 9, 115-119.

Wlazlo, Z., Hartwing, K. S., Hand, I., Kaiser, G., \& Münchau, N. (1990). Exposure in vivo vs social skills training for social phobia: Long-term outcome and differential effects. Behavior Research and Therapy, 28, 181-193.

Wolpe, J. (1977). Inadequate behavior analysis: The Achilles hell of outcome research in behavior therapy. Journal of Behavior Therapy and Experimental Psychiatry, 8, 1-3.

Wolpe, J. (1981). The dichotomy between classical conditioned and cognitively learned anxiety. Journal of Behavior Therapy and Experimental Psychiatry, 12, 35-42.

Woods, D. W., \& Miltenberger, R. G. (1996). Are persons with nervous habits nervous? A preliminary examination of habits function in a nonreferred population. Journal of Applied Behavior Analysis, 29, 259-261. 